\title{
Optical coherent transients in cold atoms: From free-induction decay to optical precursors
}

\author{
J. F. Chen, Shuyuan Wang, Dong Wei, M. M. T. Loy, G. K. L. Wong, and Shengwang Du* \\ Department of Physics, The Hong Kong University of Science and Technology, Clear Water Bay, Kowloon, \\ Hong Kong, People's Republic of China \\ (Received 4 December 2009; revised manuscript received 10 January 2010; published 26 March 2010)
}

\begin{abstract}
We report theoretical and experimental studies of the propagation of a square-modulated laser pulse through a laser-cooled atomic ensemble of two-level absorbers, as well as through a three-level system with electromagnetically induced transparency. We find that the transmission characteristics over a wide range of optical depth can be fully accounted for as optical coherent transients excited by the wide spectral content of the square-modulated optical pulse. We show that both time-domain atom-field coupled equations and frequency-domain linear dispersion theory give precisely the same description of the optical transients at a weak power limit. At low optical depth with moderate absorption, resonant excitation dominates and free-induction decay (FID) contributes mostly to the transient field. At high optical depth when absorption and propagation effects become significant, lossless optical precursors start to dominate the transient response. By varying the optical depth from 0 to 45 , we observe that optical transients evolve gradually from FID to optical precursors. We thus show that FID and optical precursors, which have been considered as two different optical transients for many decades, can be unified within a single theoretical frame and they are the manifestations of the same physical process in two different regimes.
\end{abstract}

DOI: 10.1103/PhysRevA.81.033844

PACS number(s): 42.50.Md, 42.50.Gy, 42.25.Bs

\section{INTRODUCTION}

The interaction of radiation with matter is at the heart of the research field of atomic, molecular, and optical physics, with the radiation field described by Maxwell's wave equation and the matter system described by quantum mechanics, coupled through the electromagnetic (EM) interaction. From early times on, rather than seeking a general solution, various approximations have been applied as appropriate to the physical system at hand. The theory of EM wave propagation in matter was developed around 1900 based on approximating the material system as a collection of linear oscillators [1]. With the material part approximated by a dispersive linear response function, the Maxwell wave equation can be solved. Further, with the Fourier technique (and within this linear approximation), both steady-state and transient propagating solutions can be obtained. Along this line, the concept of optical precursors emerged from the theoretical study of Sommerfeld and Brillouin on causality in EM wave propagation in dispersive media near resonance in 1914 [2,3]. They concluded that for a sufficiently fast input pulse, a transient forerunner, now known as Sommerfeld-Brillouin (SB) precursor, travels with the velocity of light in vacuum, followed by the main pulse traveling at group velocity. Optical precursors have been studied extensively both theoretically [4-9] and experimentally [10-14]. Precursor behaviors have been reported in $\gamma$ rays [15], microwaves [16], sound [17,18], optical waves [10-12], and single-photon correlations [13,14]. However, in these previous experiments, while the observed features are in qualitative agreement with theory, those data were rather limited in both scope and precision, far below what is required to compare with theoretical predictions. Because of the lack of a clear signature, some researchers do not

\footnotetext{
*dusw@ust.hk
}

believe that optical precursors have been observed [19-21]. Most recently, making use of electromagnetically induced transparency (EIT) and the slow-light effect [22,23] in cold atoms with high optical depth, we successfully generated and separated the optical precursor from a delayed main field [24].

On the other hand, the phenomena of optical nutation and free-induction decay (FID) were studied extensively in the early days after the invention of the laser [25-30], probably because of the realization that the equations governing such a system are formally equivalent to the well-studied spinresonance Bloch equation [31,32]. Recently, optical FID with long coherence time generated using cold atoms has been reported [33,34]. Measuring these optical coherent transients in the time domain has become an important approach in studying atomic and molecular spectroscopy which are complementary to those made in the frequency domain [35].

However, the two phenomena, optical precursors and FID, have generally been regarded as two separate effects in the past decades. There have been many attempts to unify the concepts of optical precursors and coherent transients. Avenel et al. [36] have pointed out that Crisp's coherent optical transients of $0 \pi$ pulse [37] can be explained in terms of optical precursors, but without mathematical justification. Recently, Jeong and Österberg showed that precursors and $0 \pi$ pulses can be unified [38]. Later, LeFew et al. provided an accurate description of optical precursors and their relation to coherent optical transients and theoretically proved that Crisp's $0 \pi$ pulses are indeed optical precursors [9]. In this article, we report a detailed experimental and theoretical investigation of the propagation of a square-modulated laser pulse through a laser-cooled atomic ensemble in a two-level system as well as in a three-level system with EIT. The data obtained over a wide range of optical depth (from 0 up 45) show that at low optical depth, the transmitted wave can be described as FID 
and at high optical depth as optical precursors. We show that our data at low optical power limit can be fully accounted for by theories of time-domain atom-field coupled equations, as well as the frequency-domain linear dispersion approach.

The article is organized as follows: In Sec. II, we provide a general theoretical formalism to describe optical coherent transients in the time and frequency domains. Section III describes the experiment, from the experimental setup (Sec. III A) to observation of FID at low optical depth (Sec. III B) and optical precursors at high optical depth (Sec. III C). In Sec. III D, we report the observation of the evolution from FID to precursor by varying the optical depth. At last we give our conclusion in Sec. IV.

\section{GENERAL THEORETICAL FORMALISM}

The theoretical frameworks that have been used to describe optical FID and precursors appear to be quite different, which is natural because the two phenomena occur under very different conditions of optical thickness and excitation strength. FID focuses on the study of atomic dynamics, aiming for a deeper understanding of quantum interaction between light and matter. The theoretical description of these optical phenomena naturally followed the optical Bloch equations [32], where the focus is on the time-dependent phase coherence of the two states excited by the radiation. Linear approximation is not made and the theory is valid for strong fields. The result is particularly simple for optically thin samples when propagation effects can be neglected. On the other side, the study of propagation patterns of the wave, SB precursors, aims for insights in nonviolation of causality. Due to this difference in focus, the relation between FID and precursors remains unclear, and they are generally regarded as two unrelated optical transient effects occurring in very different physical regimes. With the advent of cold atom traps and tunable diode lasers, we now have a single physical system with parameters that can be widely tuned to cover both physical regimes. In the following we give a detailed account of the calculations we have done using two different theoretical approaches. We show that these two types of coherent transients can be unified within a single theoretical frame and they are the manifestations of the same physical process in two different regimes.

\section{A. Time-domain atom-field coupled equations}

Optical field propagation is governed by Maxwell's equations coupled with light-matter interactions. In this article, we consider an optical pulse passing through an EIT three-level $\Lambda$ system as shown in Fig. 1. A continuous-wave (cw) coupling laser $\left(\omega_{c}\right)$ is applied on resonance to the transition $|2\rangle \rightarrow|3\rangle$ and render the medium transparent for otherwise absorption resonance transition $|1\rangle \rightarrow|3\rangle$. A probe laser pulse with a carrier angular frequency $\omega_{p}$ is applied to the transition $|1\rangle \rightarrow|3\rangle$ with a detuning of $\Delta \omega_{p}=\omega_{p}-\omega_{31}$. We describe the probe laser (real) field with the following notation:

$$
\mathbf{E}_{\mathbf{p}}(z, t)=\frac{1}{2} E_{p}(z, t) e^{i\left[k_{p 0} z-\omega_{p} t\right]}+\text { c.c. },
$$

where $k_{p 0}=\omega_{p} / c$ is the carrier wave number in vacuum. $E_{p}(z, t)$ is the slowly varying complex field envelope. The atomic motion driven by the coupling and probe lasers is

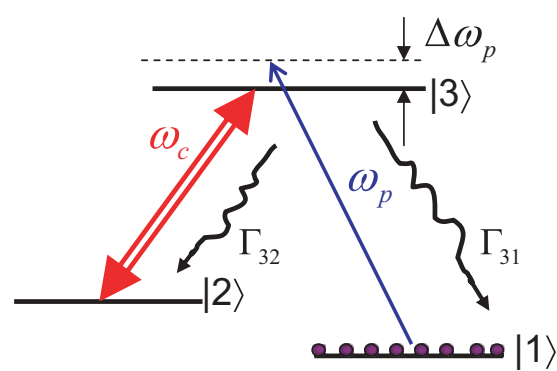

FIG. 1. (Color online) Schematic of an EIT $\Lambda$ system. $\omega_{p}$ and $\omega_{c}$ are the optical angular frequencies of the probe beam and coupling beam, respectively. $\Gamma_{31}$ and $\Gamma_{32}$ denote the atomic population decay rate of the transitions $|3\rangle \rightarrow|1\rangle$ and $|3\rangle \rightarrow|2\rangle$, respectively. $\Delta \omega_{p}$ is the detuning of the probe beam from the transition $|1\rangle \rightarrow|3\rangle$.

governed by the following master equations in the rotating reference frame:

$$
\begin{gathered}
\frac{\partial \rho_{11}}{\partial t}=-\frac{i}{2} \rho_{13} \Omega_{p}+\frac{i}{2} \rho_{31} \Omega_{p}^{*}+\Gamma_{31} \rho_{33} \\
\frac{\partial \rho_{22}}{\partial t}=-\frac{i}{2} \rho_{23} \Omega_{c}+\frac{i}{2} \rho_{32} \Omega_{c}{ }^{*}+\Gamma_{32} \rho_{33} \\
\frac{\partial \rho_{21}}{\partial t}=i\left(\Delta \omega_{p}+i \gamma_{12}\right) \rho_{21}+\frac{i}{2} \rho_{31} \Omega_{c}^{*}-\frac{i}{2} \rho_{23} \Omega_{p} \\
\frac{\partial \rho_{23}}{\partial t}=-\gamma_{23} \rho_{23}+\frac{i}{2}\left(\rho_{33}-\rho_{22}\right) \Omega_{c}^{*}-\frac{i}{2} \rho_{21} \Omega_{p}^{*} \\
\frac{\partial \rho_{31}}{\partial t}=i\left(\Delta \omega_{p}+i \gamma_{13}\right) \rho_{31}+\frac{i}{2} \rho_{21} \Omega_{c}-\frac{i}{2}\left(\rho_{33}-\rho_{11}\right) \Omega_{p} \\
1=\rho_{11}+\rho_{22}+\rho_{33}
\end{gathered}
$$

where $\rho_{i j}=\langle i|\hat{\rho}| j\rangle$ is the atomic density matrix element and we have applied the relation $\rho_{j i}=\rho_{i j}^{*} ; \Omega_{p}(z, t)=$ $E_{p}(z, t) \mu_{31} / \hbar$ is the complex Rabi frequency of the probe laser; $\Omega_{c}=E_{c} \mu_{32} / \hbar$ is the coupling laser Rabi frequency, where the constant $E_{c}$ is the electric field envelope of the cw coupling laser; $\mu_{i j}$ is the electric dipole matrix element; and $\gamma_{i j}$ is the dephasing rate between $|i\rangle$ and $|j\rangle$. The population decay rate from $|3\rangle$ to $|1\rangle$ and $|2\rangle$ are $\Gamma_{31}$ and $\Gamma_{32}$, respectively. The total decay rate of atoms in the excited state $|3\rangle$ is thus $\Gamma_{3}=\Gamma_{31}+\Gamma_{32}$. For cold atoms, we take $\gamma_{13}=\gamma_{23}=\Gamma_{3} / 2$.

When the coupling laser is off $\left(\Omega_{c}=0\right)$ and $\Gamma_{32}=0$, Eqs. (2) reduce to the well-known Bloch equations for a closed two-level system,

$$
\begin{gathered}
\frac{\partial W_{31}}{\partial t}=2 \operatorname{Im}\left\{\rho_{31} \Omega_{p}^{*}\right\}-\Gamma_{3}\left(1+W_{31}\right) \\
\frac{\partial \rho_{31}}{\partial t}=i\left(\Delta \omega_{p}+i \gamma_{13}\right) \rho_{31}-\frac{i}{2} \Omega_{p} W_{31},
\end{gathered}
$$

where $W_{31}=\rho_{33}-\rho_{11}$ is the population inversion.

Under the slowly varying envelope approximation (SVEA), the probe laser propagation can be described by a reduced wave equation,

$$
\frac{\partial E_{p}(z, t)}{\partial z}+\frac{1}{c} \frac{\partial E_{p}(z, t)}{\partial t}=\frac{i}{2} \frac{\omega_{p}}{c \varepsilon_{0}} P(z, t),
$$


where $P(z, t)=2 N \mu_{13} \rho_{31}(z, t)$ is the induced electric dipole moment complex envelope in the probe transition with atomic density $N$.

One can obtain the probe field output by solving Eqs. (2)-(4) under the initial and boundary conditions $\hat{\rho}(z, t=0)=|1\rangle\langle 1|$ and $E_{p}(z=0, t)=E_{0}(t)$. The configuration shown in Fig. 1 allows us to study both EIT and two-level systems in a wide parameter space. In this article, we will use these equations to numerically simulate both FID and optical precursors.

The preceding three-level atom-field coupled equations in time-space domain are generally nonlinear and can only be solved numerically. However, in a medium of two-level atoms, the nonlinear responses, such as saturation and nutation effects, can be solved using the well-known vector model under the adiabatic-following approximation $[39,40]$. To explore the physics behind the preceding equations, in the following, we discuss FID excited by a weak step-modulated probe pulse through a two-level system at low optical depth. An ideal step-on (-off) pulse envelope with amplitude $E_{0}$ can be described by a heaviside function $E_{0 \pm}(t)=E_{0} \Theta( \pm t)$, where "+" denotes the step-on pulse with a rising edge and "-" for the step-off pulse with a falling edge.

At first, we consider the step-off case $E_{0} \Theta(-t)$. When the probe field is sufficiently weak, almost all the atomic population is at the ground state $|1\rangle$; that is, $W_{31} \simeq-1$. Under this approximation, from Eq. (3) we obtain an evolution equation for $\rho_{31}$ :

$$
\frac{\partial \rho_{31}}{\partial t} \simeq i\left(\Delta \omega_{p}+i \gamma_{13}\right) \rho_{31}+\frac{i}{2} \Omega_{p}
$$

At $t=0$, the steady-state value of $\rho_{31}$ can be solved from $\partial \rho_{31} / \partial t=0$ :

$$
\rho_{310}=\frac{-\Omega_{p} / 2}{\Delta \omega_{p}+i \gamma_{13}} .
$$

For $t>0$, we turn off the external probe laser field and set Eq. (6) as the initial condition. Solving Eq. (5), we obtain

$$
\rho_{31}(t)=\rho_{310} \Theta(t) e^{i \Delta \omega_{p} t} e^{-\gamma_{13} t},
$$

and the complex envelope of electric polarization,

$$
\begin{aligned}
P_{0}(t) & =2 N \mu_{13} \rho_{31}(t) \\
& =\frac{-\varepsilon_{0} \alpha_{0} \gamma_{13}}{k_{p 0}\left(\Delta \omega_{p}+i \gamma_{13}\right)} E_{0} \Theta(t) e^{i \Delta \omega_{p} t} e^{-\gamma_{13} t}
\end{aligned}
$$

where $\alpha_{0}=2 N k_{p 0}\left|\mu_{13}\right|^{2} /\left(\varepsilon_{0} \hbar \Gamma_{3}\right)$ is the on-resonance absorption coefficient. Therefore, after the external probe laser is switched off, the induced atomic electric dipoles do not disappear immediately. The exponential decay is caused by the dephasing process. The FID field at the medium output surface $(z=L)$ results from the coherent radiation of the freely decayed electric dipole. At low optical depth, the induced electric dipoles (envelope) are spatially in phase and their propagation effect can be ignored. In this case, the probe laser field can be approximated as $E_{p}(z, t>0) \simeq E_{\mathrm{FID}}(z, t)$, where the FID field satisfies

$$
\frac{\partial}{\partial z} E_{\mathrm{FID}}(z, t) \simeq \frac{i k_{p 0}}{2 \varepsilon_{0}} P_{0}(t),
$$

which gives the solution of the FID field envelope at the output

$$
\begin{aligned}
E_{\mathrm{FID}}(L, t) & =\frac{i k_{p 0} L}{2 \varepsilon_{0}} P_{0}(t) \\
& =-i \frac{\alpha_{0} L}{2} \frac{\gamma_{13} E_{0}}{\Delta \omega_{p}+i \gamma_{13}} \Theta(t) e^{i \Delta \omega_{p} t} e^{-\gamma_{13} t} .
\end{aligned}
$$

This is the electric field envelope of FID after the probing beam is suddenly removed. The decay term $e^{-\gamma_{13} t}$ is intrinsic to the atomic system itself. At the limit of $\alpha_{0} L \ll 1$, Eq. (10) gives us a good approximation to the induced electric field because nearly no absorption occurs. In this limit, the field amplitude of FID is proportional to the optical depth $\alpha_{0} L$ due to the collective enhancement. To take into account the finite absorption, Eq. (10) can be more precisely given by multiplying a factor of $e^{-\alpha_{0} L / 4}$ [see Appendix A]:

$$
E_{\mathrm{FID}}(L, t)=-i \frac{\alpha_{0} L}{2} \frac{\gamma_{13} E_{0}}{\Delta \omega_{p}+i \gamma_{13}} e^{-\alpha_{0} L / 4} \Theta(t) e^{i \Delta \omega_{p} t} e^{-\gamma_{13} t}
$$

The physics picture of the factor $e^{-\alpha_{0} L / 4}$ is clear: On average, the FID field experiences half of the absorption length because and we can consider that the induced electric dipole is effectively located at the center of the medium. With $\Delta \omega_{p}=$ $\omega_{p}-\omega_{31}$, the central optical frequency of the FID field after the falling edge is always on atomic resonance $\left(\omega_{31}\right)$ for a weak power excitation and does not depend on the frequency of the driving field.

For the step-on pulse with a rising edge $E_{0+}(t)=E_{0} \Theta(t)$, with the the relation $\Theta(t)=1-\Theta(-t)$, we obtain the output field

$$
E_{+}(t)=\bar{E} \Theta(t)-E_{\mathrm{FID}}(L, t) .
$$

The first term is the steady-state value, and the second is the same as the FID field from a step-off pulse but with an opposite sign. Therefore, at the rising edge, when the absorption is not significant, there is interference between the FID and the steady-state carrier field.

The preceding approximations are valid for optical coherent transients excited by a weak step-modulated optical pulse in an optically thin medium where the FID signal reveals the revolution of the induced electric dipole. When the optical depth $\left(\alpha_{0} L\right)$ is large, the propagation effect cannot be ignored.

\section{B. Frequency-domain linear dispersion theory}

When the probe laser is sufficiently weak, the atomic population remains mostly in the ground state $|1\rangle$. Under such a ground-state approximation, the excitation from the Fourier frequency components of the probe pulse can be treated independently and nonlinear wave mixing between them can be ignored in the first-order perturbation theory. In this section, with frequency-domain linear dispersion approach, we will show that both FID and the optical precursor can be derived from the same Fourier integral.

The input probe pulse (field envelope) $E_{0}(t)$ at $z=0$ can be decomposed in to a continuous spectrum in the Fourier frequency domain:

$$
E_{0}(t)=\frac{1}{2 \pi} \int E_{0}(\omega) e^{-i \omega t} d \omega .
$$


Here $\omega$ should be understood as the deviation from the probe carrier angular frequency. Under the condition of weak probe power and ground-state approximation, the atom-field coupled equations (2)-(4) in Sec. II can be linearized in the frequency domain. Solving the linearized Eq. (2) using the Fourier transform, the induced electric dipole in the frequency domain at the probe transition is then determined by the following linear relation [23]:

$$
P(\omega)=\varepsilon_{0} \chi(\omega) E(\omega) .
$$

The EIT linear susceptibility (complex) can be obtained as

$$
\chi(\omega)=\frac{\alpha_{0}}{k_{p 0}} \frac{4\left(\omega+\Delta \omega_{p}+i \gamma_{12}\right) \gamma_{13}}{\left|\Omega_{c}\right|^{2}-4\left(\omega+\Delta \omega_{p}+i \gamma_{12}\right)\left(\omega+\Delta \omega_{p}+i \gamma_{13}\right)} .
$$

As we turn off the coupling laser, that is, $\Omega_{c}=0$, Eq. (15) reduces to that of a two-level system:

$$
\chi(\omega)=\frac{\alpha_{0}}{k_{p 0}} \frac{-\gamma_{13}}{\omega+\Delta \omega_{p}+i \gamma_{13}} .
$$

Then the propagation of the weak probe field envelope is given by the integral

$$
\begin{aligned}
E(L, t) & =\frac{1}{2 \pi} \int E_{0}(\omega) e^{i[\Delta k(\omega) L-\omega t]} d \omega \\
& =\frac{1}{2 \pi} \int E_{0}(\omega) H(\omega) e^{-i \omega t} d \omega,
\end{aligned}
$$

where $\quad \Delta k=k-k_{p 0} \cong \frac{\omega}{c}+\frac{1}{2} k_{p 0} \chi(\omega) \cong \frac{1}{2} k_{p 0} \chi(\omega)$ and $H(\omega)=e^{i \Delta k(\omega) L}$ is the medium linear transfer function.

Now we derive the FID from the frequency-domain linear dispersion theory. At low optical depth, we approximate the medium transfer function as

$$
H(\omega) \simeq 1+\frac{i k_{p 0} L}{2} \chi(\omega)
$$

which connects to its impulse response from a two-level system in time domain,

$$
\begin{aligned}
h(t) & =\frac{1}{2 \pi} \int H(\omega) e^{-i \omega t} d \omega \\
& \simeq \delta(t)-\frac{\alpha_{0} L \gamma_{13}}{2} \Theta(t) e^{i \Delta \omega_{p} t} e^{-\gamma_{13} t} .
\end{aligned}
$$

With the input step-off pulse $E_{0-}(t)=E_{0} \Theta(-t)$, the total field output can be obtained from a convolution:

$$
\begin{aligned}
E(L, t) & =E_{0} \Theta(-t) * h(t) \\
& \simeq \bar{E} \Theta(-t)-i \frac{\alpha_{0} L}{2} \frac{\gamma_{13} E_{0}}{\Delta \omega_{p}+i \gamma_{13}} \Theta(t) e^{i \Delta \omega_{p} t} e^{-\gamma_{13} t} \\
& =\bar{E} \Theta(-t)-i \frac{k_{p 0} L}{2} \chi(0) E_{0} \Theta(t) e^{i \Delta \omega_{p} t} e^{-\gamma_{13} t} .
\end{aligned}
$$

The first term is the steady-state value before the external field is turned off $(t<0)$, and the second term is the same as the FID field in Eq. (10). Therefore, in the viewpoint of frequency domain, FID is indeed a linear effect excited by a weak step pulse passing through the medium.

At high optical depth, the approximation (18) cannot be applied. In this regime, the near- and on-resonance frequency components are heavily absorbed and the precursor behavior dominates the transient response. The theory of optical precursors was initiated by Sommerfeld and Brillouin in 1914 to study the causality of pulse propagation in a classical Lorentz dielectric material consisting of a collection of linear oscillators [2]. It has been well known that the linear optical properties of the classical Lorentz oscillator model are identical to those of the two-level system in quantum mechanics [41]. When the probe laser detuning is large $\left(\Delta_{p}+\omega \gg \Omega_{c}\right)$ or the coupling laser is off $\left(\Omega_{c}=0\right)$, the EIT linear susceptibility (15) reduces to Eq. (16) of the two-level system. It can be shown that, in the optical regime of our interest, taking the valid approximation $\omega_{p}+\omega+\omega_{31} \approx 2\left(\omega_{p}+\omega\right) \approx 2 \omega_{31}$ (where $\omega \ll \omega_{p}$ is the probe envelope spectrum, and $\left.\omega_{p}=\omega_{31}+\Delta \omega_{p}\right)$, the linear susceptibility in Eq. (16) of the two-level system is equivalent to the Lorentz form,

$$
\chi(\omega) \simeq \frac{-\varpi_{p l}^{2}}{\left(\omega_{p}+\omega\right)^{2}-\omega_{31}^{2}+2 i \gamma_{13}\left(\omega_{p}+\omega\right)},
$$

where $\varpi_{p l}=\sqrt{2 \alpha_{0} c \gamma_{13}}$ is the effective plasma frequency. Therefore, our system is suitable for testing the Sommerfeld and Brillouin precursor theory.

We take the stationary phase method [42] to explain the physics of precursors. With the phase $\phi=\Delta k(\omega) L-\omega t$ in Eq. (17), $e^{i \phi}$ is oscillatory at high optical depth because the phase $\phi$ changes rapidly as the frequency changes. Therefore, the dominant contribution to the integral occurs at the stationary-phase frequencies $\omega_{d}$, where $\phi^{\prime}\left(\omega_{d}\right)=d \phi / d \omega=0$. This stationary phase condition is equivalent to $L / V_{g}\left(\omega_{d}\right)=t$; that is, the dominant contribution of the field at time $t$ are the frequency components arriving at the time $t$ equal to their group delay. At time $t=L / c$, when the rising edge occurs, the infinite far-off-resonance frequencies contribute the most because they are not coupled to the atoms and travel at the speed of light in vacuum $c$. This "forerunner" transient wave following the leading edge is called a "precursor." In general, with loss present, the phase $\phi$ becomes a complex function and the stationary phase approximation is extended to the modern asymptotic method [4]. H. Jeong and S. Du developed a hybrid-asymptotic analysis to describe the precursor field and the main signal through an EIT system [7]. The main idea was to divide the pulse response into two spectral ranges and treat them separately. In the far-off-resonance regime, the precursor is derived using asymptotic analysis. In the central narrow EIT window, the main field is obtained using an impulse-response method to avoid the singularity. Similar treatment can also be found in Ref. [8].

For simplification, we consider here the probe laser is on resonance $\left(\Delta \omega_{p}=0\right)$. Given the step-on or step-off input pulse, $E_{0}(t)=E_{0} \Theta( \pm t)$, following the asymptotic analysis used in Refs. [7-9,15,17], one can obtain an analytical expression for the SB precursor [24],

$$
E_{S B \pm}(t) \simeq \pm E_{0} J_{0}\left(\sqrt{2 \alpha_{0} L \gamma_{13} \tau}\right) \Theta(\tau) e^{-\gamma_{13} \tau},
$$

where $\tau=t-L / c$.

Now we turn to the on-resonance main field. For a two-level system, the main field is absorbed at high optical depth. For an EIT system, the delayed main field cannot be obtained using the same asymptotic method [7,14]. When the ground-state dephasing is negligible $\left(\gamma_{12} \approx 0\right.$ or $\left.\left|\Omega_{c}\right|^{2} \gg 4 \gamma_{12} \gamma_{13}\right)$ and the EIT transparency window is very narrow, we can extend the 


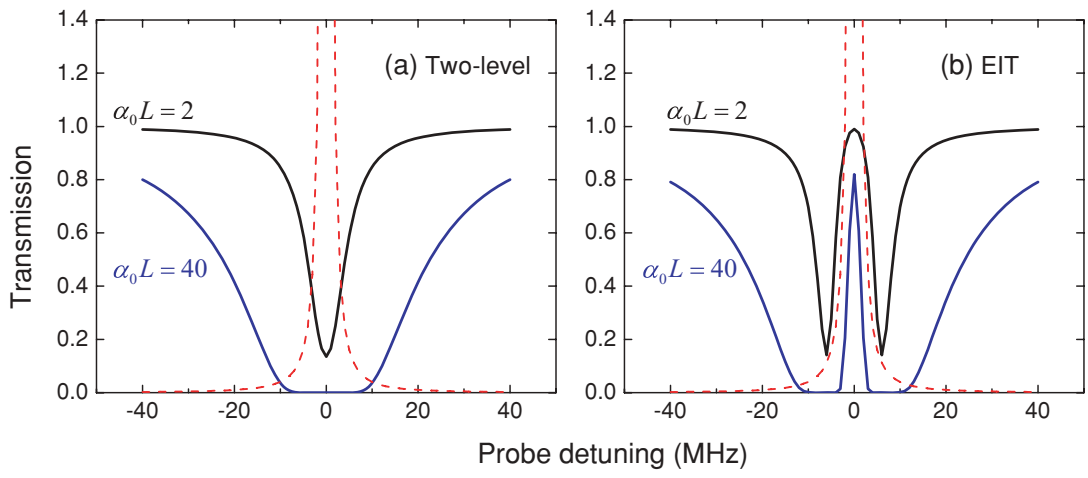

FIG. 2. (Color online) Transmission spectrum of the probe laser in (a) a two-level system $\left(\Omega_{c}=0\right)$ and (b) an EIT system at different optical depths. The red dashed lines denote the spectrum of an input step pulse.
EIT linear susceptibility [Eq. (15)] up to the third order about $\omega$ and obtain the main field as [see Appendix B]

$$
E_{M \pm}(t) \simeq \frac{E_{0}}{2} e^{-\gamma_{12} \tau_{g}}\left(1 \pm \operatorname{erf}\left[\frac{a\left(t-\tau_{g}\right)}{\sqrt{2}}\right]\right) * b \operatorname{Ai}(-b t)
$$

where $\tau_{g} \simeq 2 \alpha_{0} L \gamma_{13} /\left|\Omega_{c}\right|^{2}$ is the group delay, $a=$ $\sqrt{\alpha_{0} L} /\left(2 \tau_{g}\right)$ determines the EIT bandwidth, $b=$ $\left|\Omega_{c}\right|^{2}\left[24 \alpha_{0} L \gamma_{13}\left(\left|\Omega_{c}\right|^{2}-4 \gamma_{13}^{2}\right)\right]^{-1 / 3}$, and erf is the error function. In most cases, the effect from the Airy function is small and the main field can be approximated as [24]

$$
E_{M \pm}(t) \simeq \frac{E_{0}}{2} e^{-\gamma_{12} \tau_{g}}\left\{1 \pm \operatorname{erf}\left[\frac{a\left(t-\tau_{g}\right)}{\sqrt{2}}\right]\right\} .
$$

Then the total output field can be obtained by

$$
E_{ \pm}(t)=E_{S B \pm}(t)+E_{M \pm}(t) .
$$

In the past, FID and optical precursors have been considered as two different type of coherent transients. In the time domain, their connection is not obvious, while in the frequency domain, the linear dispersion theory provides more insights in weak probe pulse propagation through the atomic medium. Figure 2 shows the intensity transmission profiles $|H(\omega)|^{2}$ for both two-level and EIT systems. As we know, the field is strongly interacted with the atoms at their absorption resonances. For the two-level system, there is one absorption resonance, as shown in Fig. 2(a). For the EIT system in Fig. 2(b), there are two absorption resonances separated by about $\Omega_{c}$ and one transparency window between them around single-photon resonance $\omega=0$. The dashed lines represent the spectrum of a step-modulated pulse with on-resonance carrier frequency $\left(\omega_{p}=\omega_{31}\right)$. At low optical depth $\left(\alpha_{0} L\right)$, when the absorption is not significant, the on-absorption-resonance frequency components dominate. These frequency components strongly coupled to the atomic system contribute to the FID signal and dominate the optical transient response at low optical depth. At high optical depth when the on-absorption-resonance frequency components are absorbed, optical precursors start to be formed from the lossless far-detnuned frequency components that propagate nearly at the speed of light in vacuum $c$. In this case, the optical transients are dominated by optical precursors. In the two-level system, the main field is absorbed and the precursors "precede" nothing, while in the EIT system, the main field lying in the narrow transparency window is delayed due to the slow-light effect, and thus it is possible to separate the optical precursors completely from the main field [24]. For both the two extreme regimes, we have obtained analytic solutions. For the intermediate regime, we can solve the equations numerically.

We have numerically verified that, in the weak-probe-power cases, both time-domain atom-field coupled equations and frequency-domain linear dispersion theory give equivalent and precise description for optical transients in a wide parameter space, in both the EIT and the two-level systems. When the probe power becomes higher, in general, the atomic system becomes nonlinear and the frequency-domain linear dispersion theory does not work.

\section{EXPERIMENT}

\section{A. Experimental setup}

We work with cold ${ }^{85} \mathrm{Rb}$ atoms in a two-dimensional (2D) magneto-optical trap (MOT). The 2D quadruple magnetic field has a transverse gradient of $10 \mathrm{G} / \mathrm{cm}$. The experiment is run periodically with $4.5 \mathrm{~ms}$ MOT time followed by a $0.5-\mathrm{ms}$ measurement window. Just before the end of the MOT time, the repump laser is turned off $0.3 \mathrm{~ms}$ before the trapping laser to prepare all the atoms in the ground level $|1\rangle$. The atomic cloud in the 2D MOT, with a temperature of about $100 \mu \mathrm{K}$, has a length of about $L=1.5 \mathrm{~cm}$ and transverse diameter of $0.5 \mathrm{~mm}$.

Figure 3 shows the experiment configuration for measuring FID and optical precursors. We take $|1\rangle=\left|5 S_{1 / 2}, F=2\right\rangle$,

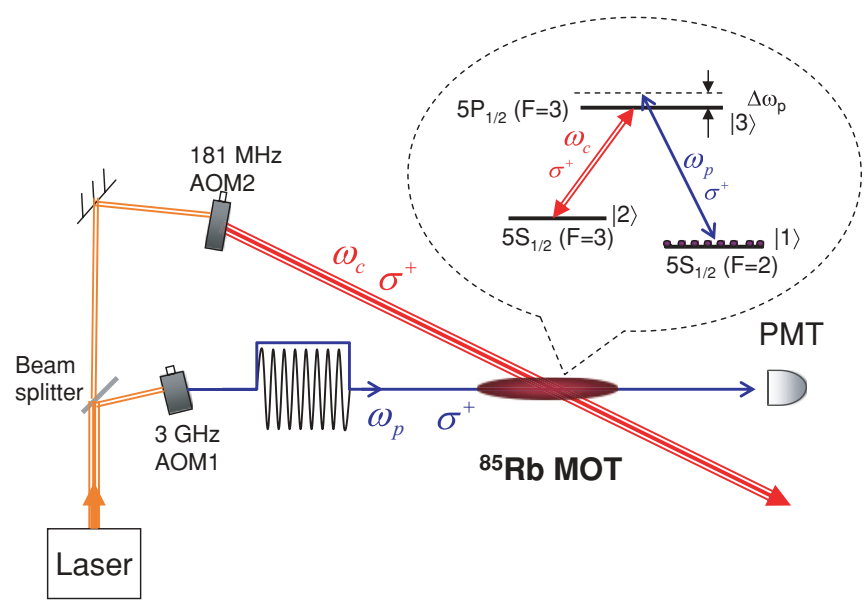

FIG. 3. (Color online) Experimental configuration for measuring optical transients in cold ${ }^{85} \mathrm{Rb}$ atoms. Both coupling and probe laser beams are directed from a single laser to eliminate EIT two-photon phase-frequency fluctuation. (Inset) EIT energy level diagram. 
$|2\rangle=\left|5 S_{1 / 2}, F=3\right\rangle$, and $|3\rangle=\left|5 P_{1 / 2}, F=3\right\rangle$ for the atomic energy level configuration. The atomic decay rates are $\Gamma_{3}=$ $2 \pi \times 6 \mathrm{MHz}, \Gamma_{31}=\frac{15}{27} \Gamma_{3}$, and $\Gamma_{32}=\frac{12}{27} \Gamma_{3}$. The lifetime determined dephasing rates are $\gamma_{13}=\gamma_{23}=\Gamma_{3} / 2$. The groundstate dephasing rate is measured to be $\gamma_{12}=0.01 \gamma_{13}$. Both coupling and probe laser beams are directed and split from a single laser operated at the ${ }^{85} \mathrm{Rb} D_{1}$ line $(795 \mathrm{~nm})$. The laser is locked to the cross-over of the transitions $\left|5 S_{1 / 2}, F=3\right\rangle \rightarrow$ $\left|5 P_{1 / 2}, F=3\right\rangle$ and $\left|5 S_{1 / 2}, F=3\right\rangle \rightarrow\left|5 P_{1 / 2}, F=2\right\rangle$ using Doppler-free saturation absorption spectropy. After a beam splitter, the beam with main laser power passes through a 181- $\mathrm{MHz}$ acousto-optic modulator (AOM) and the +1 order serves as the coupling laser that is on resonance with the transition $|2\rangle \rightarrow|3\rangle$. The probe laser is directed from a highfrequency AOM (Brimrose) with a central frequency of about $3 \mathrm{GHz}$. We can vary the AOM central frequency to change the probe laser detuning. This configuration eliminates the relative phase-frequency noise in the EIT two-photon transition. Both coupling and probe lasers are identically circularly polarized $\left(\sigma^{+}\right)$to optimize the EIT effect due to the multi-Zeeman-state effect. The weak probe laser pulse, after propagating through the MOT, is detected by a photon-multiplier tube (PMT, Hamamatsu, H6780-20) and recorded by a 1-GHz high-speed digital scope (Tektronix, TDS684B). To avoid entering into the PMT, the coupling beam was slightly deviated from the probe beam by $2^{\circ}$. The measured optical depth $\left(\alpha_{0} L\right)$ of the on-resonance probe transition can be varied from 0 to 45 .

We use the 3-GHz AOM to generate a square pulse with a length of $2 \mu \mathrm{s}$. Such a square-modulated pulse with sufficient length allows us study optical transients at both rising and falling edges in a single shot. In Sec. II, we have theoretically investigated optical transients induced by ideal step pulses. However, in experiment, the square pulses generated by the AOM have a finite rise and fall time of $7 \mathrm{~ns}$. To simplify the theoretical analysis and numerical simulation, we model a real step pulse by turning on (off) the field amplitude linearly in the finite rise (fall) time $\Delta t$. Therefore, a real square square pulse with a length $T=2 \mu \mathrm{s}$ and rise (fall) time $\Delta t=7 \mathrm{~ns}$ can be mathematically expressed as a convolution of two ideal square functions:

$$
\begin{aligned}
\widetilde{E}_{0}(t) & =\frac{E_{0}}{\Delta t} \Pi(t, T) * \Pi(t, \Delta t) \\
& =\frac{1}{\Delta t} \int_{0}^{\Delta t} E_{0} \Pi\left(t-t^{\prime}, T\right) d t^{\prime}
\end{aligned}
$$

The unit square function is defined as $\Pi\left(t, t_{1}\right)=1$ for $t \in$ $\left[0, t_{1}\right]$ and otherwise zero. The rise (fall) time of $7 \mathrm{~ns}$ is fast enough compared to the atomic dephasing time $1 /\left(2 \gamma_{13}\right)=$ $26.5 \mathrm{~ns}$ that determines the transient field duration. A typical measured square pulse (circular points) is shown in Fig. 4(a). In the following sections for the comparison between theory and experiment, Eq. (26) will be used as the input pulse envelopeblue solid line in Fig. 4(a)-for numerical simulation. In the linear regime, both time- and frequency-domain approaches give identical results. For high-probe-power case where the frequency-domain linear dispersion theory does not work, we will solve the time-domain atom-field coupled equations numerically.

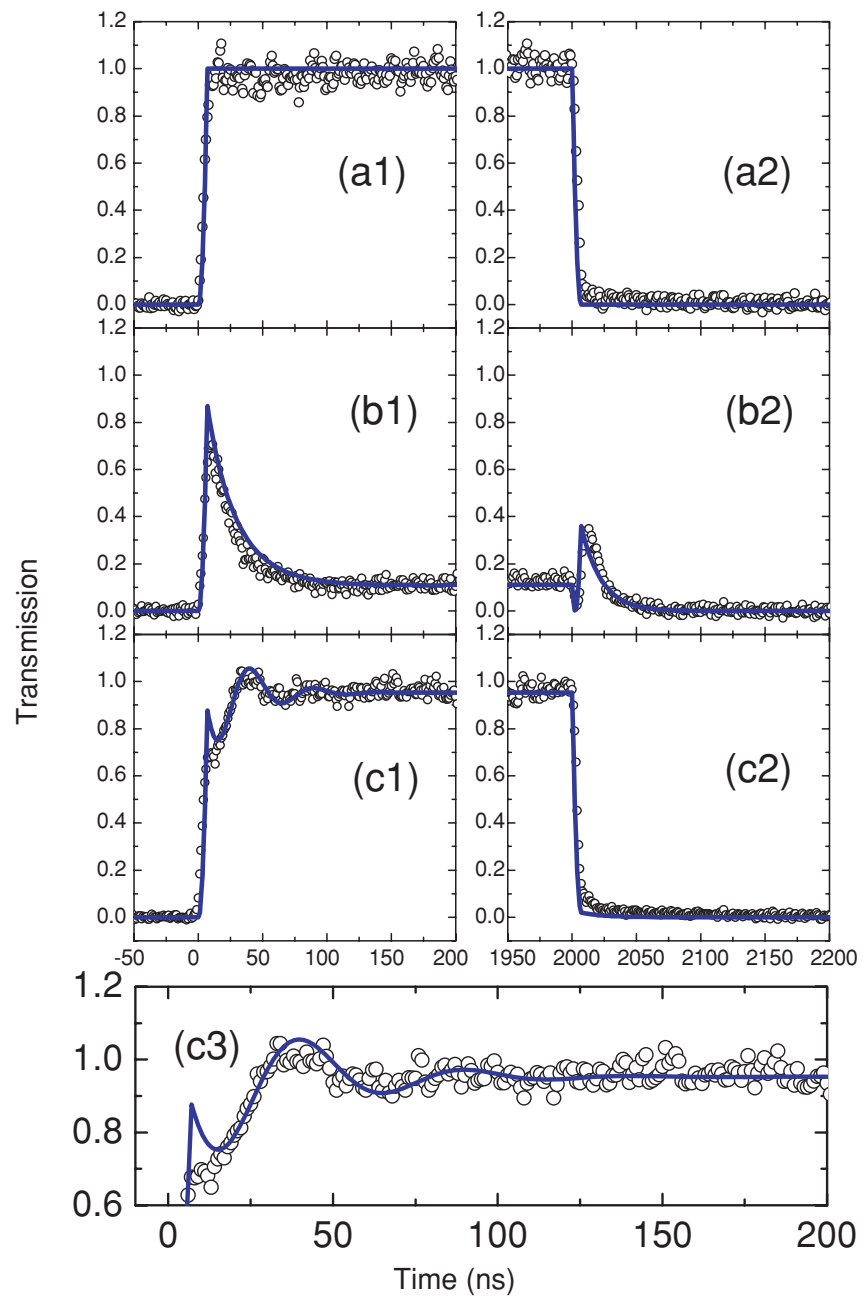

FIG. 4. (Color online) Observation of FID signals at the rising and falling edges with a weak probe pulse $\left(\Omega_{p}=0.07 \gamma_{13}\right)$. (a) The input pulse passing through the vacuum $\left(\alpha_{0} L=0\right)$. (b) $\alpha_{0} L=2.2$ and $\Delta \omega_{p}=0$. (c) $\alpha_{0} L=2.2$ and $\Delta \omega_{p}=2 \pi \times 20 \mathrm{MHz}$. Panel (c3) is an enlarged view of (c1). The black circles are experimental data. The blue solid lines are theoretical curves obtained from Eq. (17) using FFT or solving the coupled Eqs. (2) and (4).

\section{B. Free-induction decay at low optical depth}

We start measuring FID optical coherent transients excited by the weak square probe pulse $\left(\Omega_{p}=0.07 \gamma_{13}\right)$ in the twolevel system at low optical depth.

Figures 4(b1) and 4(b2) show FID signals at both rising and falling edges when the probe laser is on atomic resonance $\left(\Delta \omega_{p}=0\right)$. As expected from Eq. (10), after the falling edge, the FID intensity signal decays exponentially with a time constant of $1 /\left(2 \gamma_{13}\right)=26.5 \mathrm{~ns}$. The blue solid lines, numerically calculated from Eq. (17) using fast-Fourier transform (FFT) by taking Eq. (26) as the input pulse, agree with the experimental data very well. As already verified in Sec. II, these theoretical curves are identical to those obtained from the time-domain coupled Eqs. (2) and (4) for the weak probe pulse. From Eq. (11), the FID $E_{\mathrm{FID}}(t=0) / E_{0}=-\frac{\alpha_{0} L}{2} e^{-\alpha_{0} L / 4}=-0.63$ is in $\pi$ phase shift to the input field at the falling edge; while at the rising edge, as shown in Eq. (12), it becomes constructive interference between FID and the main steady-state field. As 


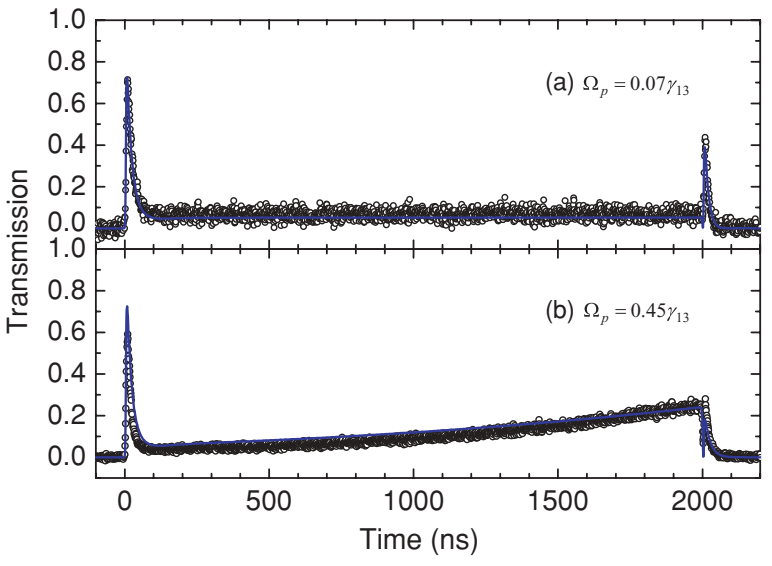

FIG. 5. (Color online) Power pumping and saturation effects in FID for the on-resonance case $\left(\Delta \omega_{p}=0\right)$. (a) $\Omega_{p}=0.07 \gamma_{13}$; (b) $\Omega_{p}=0.45 \gamma_{13} \cdot \alpha_{0} L=3$.

a result, at the rising edge, the transient signal has a much higher peak value and longer decay time constant. Figures 4(c) are results for the probe laser with off-resonance carrier frequency $\left(\Delta \omega_{p}=2 \pi \times 20 \mathrm{MHz}\right)$. Because the FID intensity signal is proportional to $\left|\Delta \omega_{p}+i \gamma_{13}\right|^{-2}$, it is too weak to be detected at the falling edge. However, at the rising edge, the FID signal is observable due to its beating with the probe carrier frequency, shown in Fig. 4(c3). The damped oscillation period, precisely determined by $2 \pi / \Delta \omega_{p}=50 \mathrm{~ns}$, confirms that the FID frequency in the weak probe case is always on atomic resonance, shown in Eq. (10). Our direct measurement of FID from a weak probe pulse clearly shows that FID can be a transient response from the linear propagation. Although the optical frequency of the FID field can be significantly different from the initial driving probe field, it is a coherent linear propagation effect, but not a spontaneous emission [43].

As we increase the probe laser power, the system becomes nonlinear. In this case, we can not apply the frequency-domain linear dispersion theory, but must solve the time-domain atom-field coupled equations (2) and (4) numerically. Figure 5 shows a comparison of the normalized optical transients with low and high probe powers at $\alpha_{0} L=3$ and $\Delta \omega_{p}=0$. There are two effects observed here. The first is the slow increase of the probe main field during the $2-\mu s$ window due to the pumping effect because the atoms slowly leak to the ground state $|2\rangle$. The second is the FID peak at the falling edge becomes smaller due to the pumping and saturation effects. The off-resonance results $\left(\Delta \omega_{p}=2 \pi \times 8 \mathrm{MHz}\right)$ are shown in Fig. 6. The theoretical curves (blue solid lines) agree with the experimental data perfectly.

\section{Optical precursor at high optical depth}

As we increase the optical depth, the near-resonance FID frequency components get absorbed strongly. At $\alpha_{0} L>10$, the transient wave patterns can be more precisely described in term of SB precursors.

Figure 7 shows the measured optical precursors (black circle points) in the two-level system $\left(\Omega_{c}=0\right)$ with $\alpha_{0} L=30$. The input weak square pulse, the same as Fig. 4(a), is on

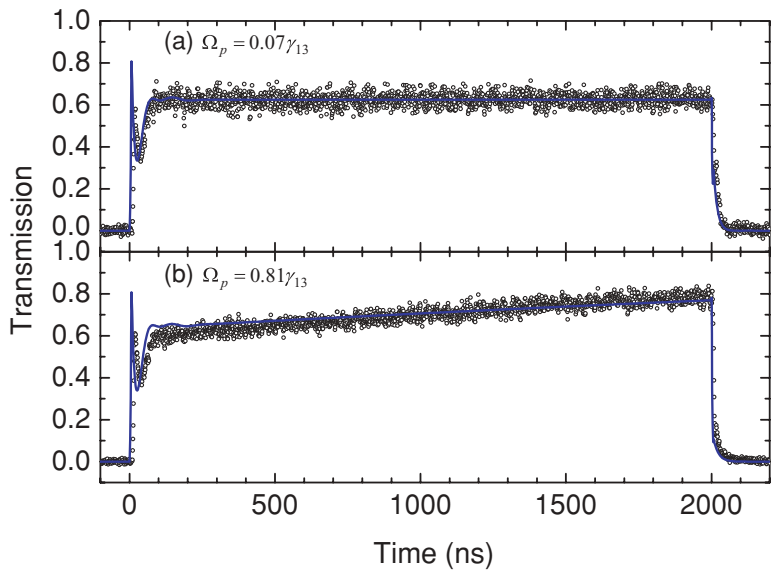

FIG. 6. (Color online) Power pumping and saturation effects in FID for the off-resonance case $\left(\Delta \omega_{p}=2 \pi \times 8 \mathrm{MHz}\right)$. (a) $\Omega_{p}=$ $0.07 \gamma_{13}$; (b) $\Omega_{p}=0.81 \gamma_{13} . \alpha_{0} L=4$.

resonance with $\Delta \omega_{p}=0$. As expected, the main field is absorbed, and the rising and falling edges show no relative time delay to that through vacuum. The blue solid lines are numerical simulations of time-space master equations (2) and (4), identical to that from Eq. (17) using FFT, and match the experimental data very well. The asymptotic results of $\left|\tilde{E}_{S B} / E_{0}\right|^{2}$ are the red dashed lines and calculated from Eq. (22) convolving with a square function $\Pi(t, \Delta t)$ to take into account the finite rise (fall) time $\Delta t=7 \mathrm{~ns}$ [Appendix B].

Figure 8 shows optical precursors in the EIT system at the same optical depth with $\Omega_{c}=4 \gamma_{13}$. At the rising edge, the transient oscillatory sparks are optical precursors identical to that observed in the two-level system. The main field is delayed by about $200 \mathrm{~ns}$ and completely separated from the

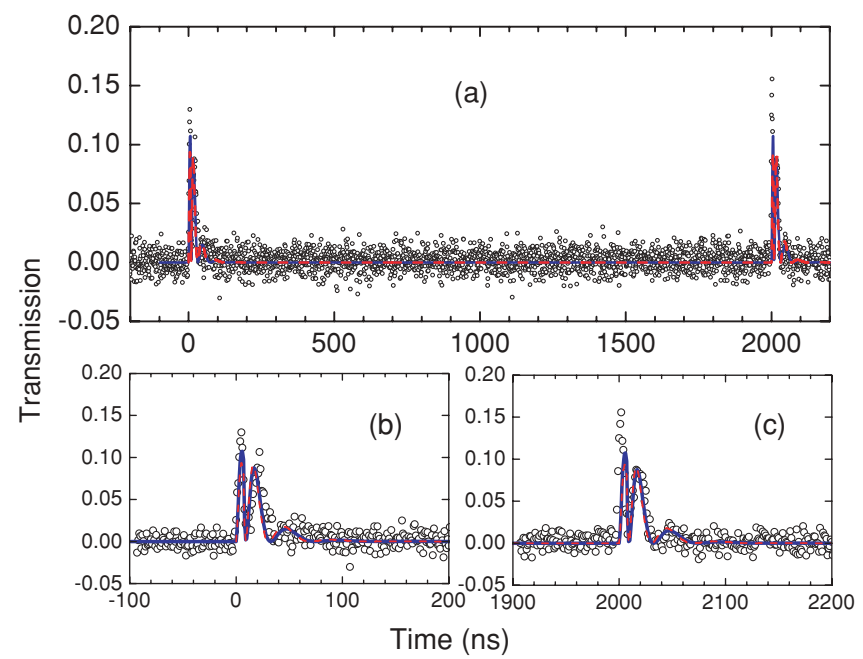

FIG. 7. (Color online) Observation of optical precursors from a weak square-modulated pulse propagating through a two-level system. $\Omega_{c}=0 ; \alpha_{0} L=30$. The black circles are experimental data. The blue solid lines are numerical simulation from Eq. (17) using FFT or solving the coupled Eqs. (2) and (4) with the input pulse the same as in Fig. 4(a). The red dash lines, overlapping with the blue solid lines, are calculated with hybrid-asymptotic analysis by taking into account the finite rise and fall time of $7 \mathrm{~ns}$. 


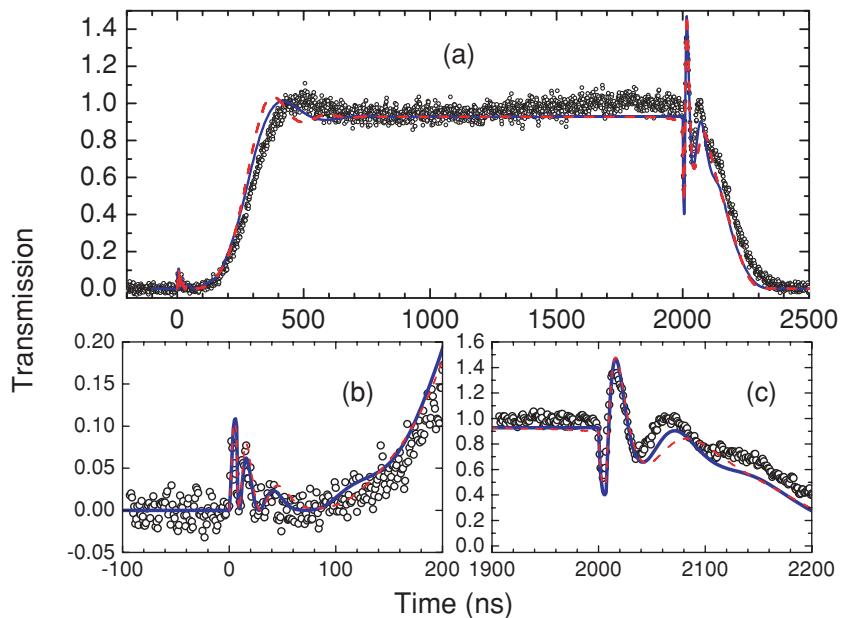

FIG. 8. (Color online) Observation of optical precursors from a weak square-modulated pulse propagating through an EIT system. $\Omega_{c}=4 \gamma_{13} ; \alpha_{0} L=30$. The black circles are experimental data. The blue solid lines are numerical simulations from Eq. (17) using FFT or solving the coupled Eqs. (2) and (4). The red dashed lines are calculated with hybrid-asymptotic analysis by taking into account the finite rise and fall time of $7 \mathrm{~ns}$.
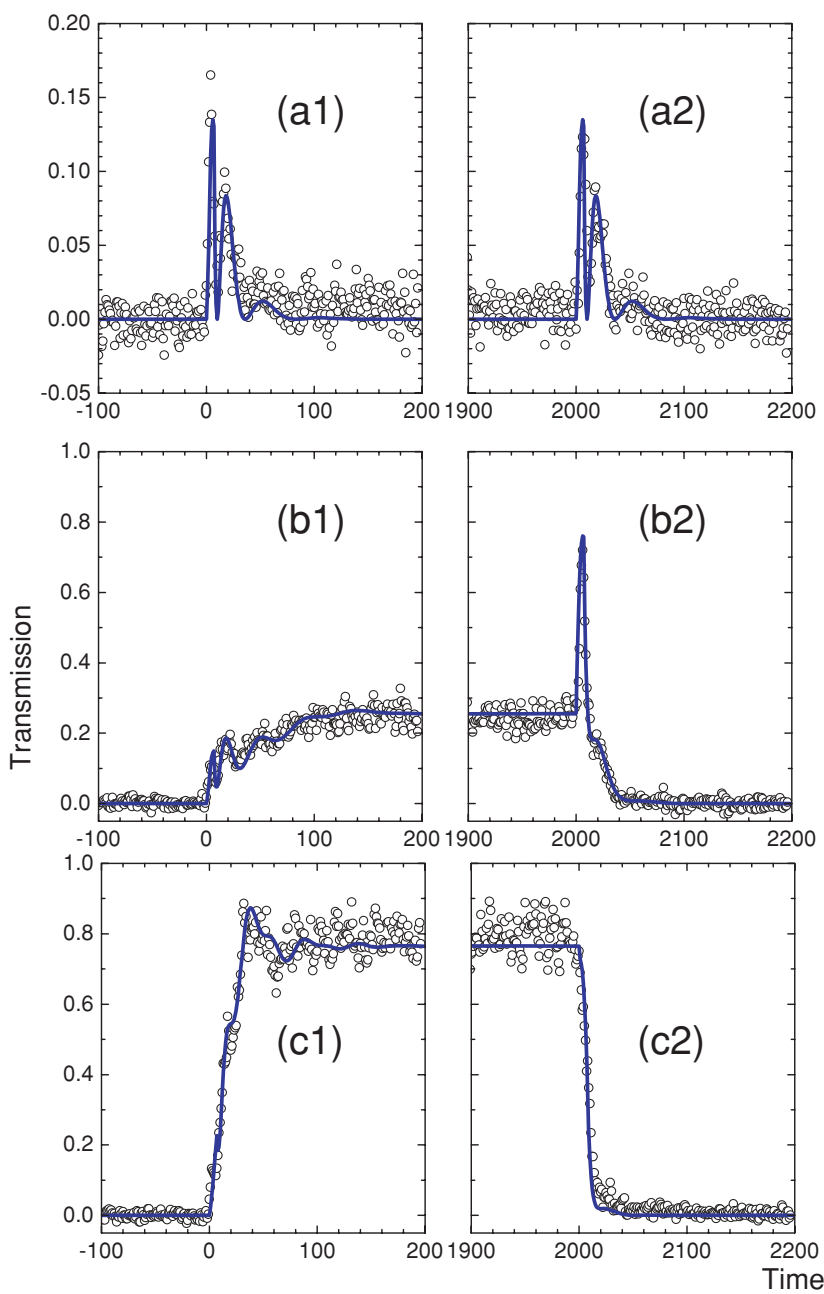

precursor. On top of the main field at about $500 \mathrm{~ns}$, we observe an oscillation feature with a long time period of about $400 \mathrm{~ns}$. This postcursor, described by a convolution with an Airy function in Eq. (23), was predicted by Macke and Segard recently [8]. At the falling edge, the oscillatory structure with a much higher peak (150\%) and longer duration results from the interference between the precursor and main field. The theoretical numerical simulations from both master equations and FFT agree well with the hybrid-asymptotic analysis and experimental data.

In the preceding discussion we focused on the case of a on-resonance weak probe pulse $\left(\Delta \omega_{p}=0\right)$. When the probe laser is detuned $\left(\Delta \omega_{p} \neq 0\right)$, the spectrum is not symmetric and it is difficult to obtain analytic solutions. For comparison, Fig. 9 shows optical transients at different probe detuning in both the two-level and the EIT systems. The theoretical curves are obtained numerically using FFT. As we increase the probe detuning, the carrier frequency moves away from the absorption lines and turns on quickly. At large detunings, both the two-level and the EIT systems give nearly identical results because the EIT transmission profile is similar to that of the two-level system at a large frequency detuning.
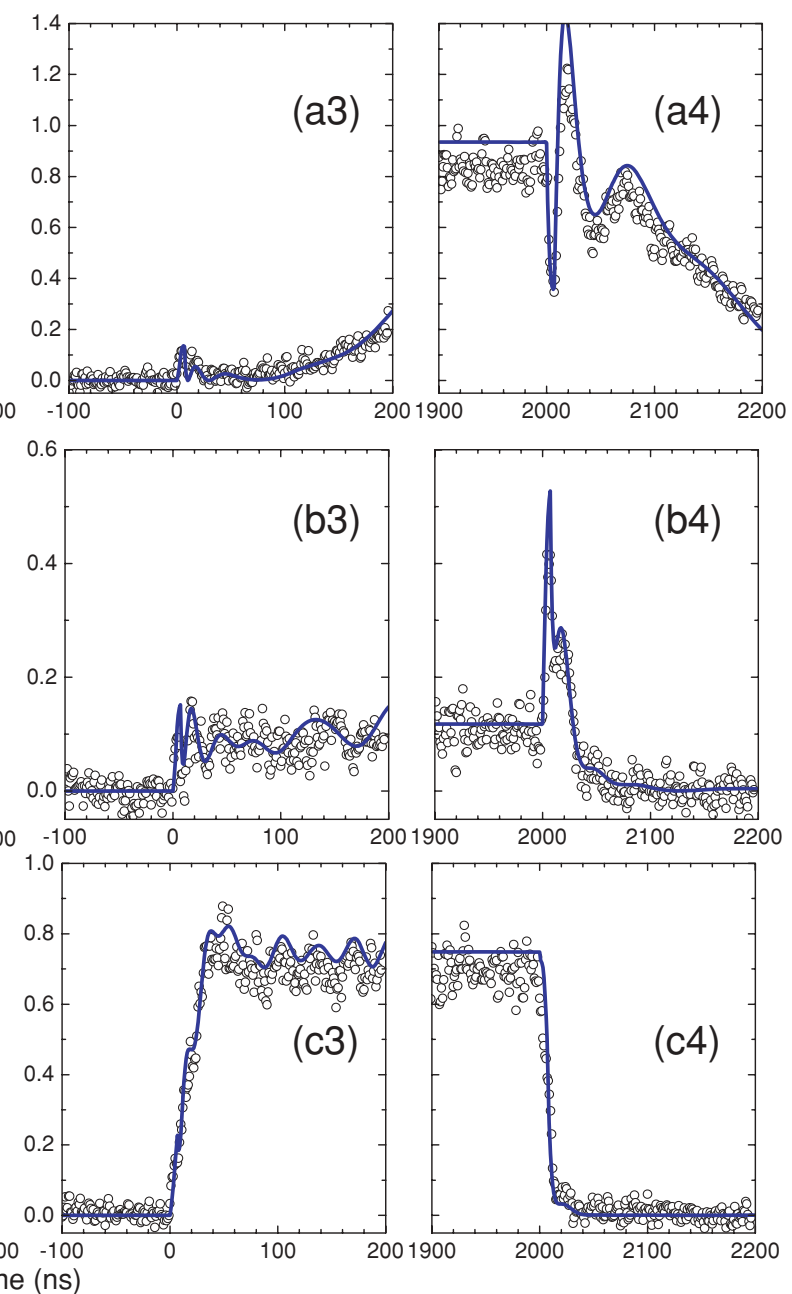

FIG. 9. (Color online) Off-resonance optical precursors measured at the two-level and EIT systems with $\alpha_{0} L=27$. Rows (a)-(c) correspond to $\Delta \omega_{p} /(2 \pi)=0,13$, and $300 \mathrm{MHz}$, respectively. Columns 1 and 2 represent the two-level system, and columns 3 and 4 represent the EIT system. 


\section{From FID to precursors}

We have studied optical transients generated from a square probe pulse through cold atoms with either low or high optical depth. In the case of low optical depth $\left(\alpha_{0} L \sim 2\right)$, the FID onresonance field dominates the transient response. In the case of high optical depth $\left(\alpha_{0} L \sim 30\right)$, the on-absorption-resonance excitations are absorbed and the far-off-resonance spectral components contribute most to optical precursors. We now turn to study the evolution from FID to precursor as we increase optical depth. For this purpose, we look at the transient peaks following the falling edge of the weak on-resonance square pulse through the two-level system where the transient field is always separated from the main steady field.

The experimental data and theoretical curve are shown in Figs. 10. To see the physics behind the curves, we need to understand the effect of finite rise and fall time of a real step pulse. In time domain, as shown in Eq. (26), it is a convolution of the ideal step pulse and a short square pulse with a length of $\Delta t$. In the frequency domain, the finite rise and fall time acts as a low-pass filter with a cut frequency proportional to $1 / \Delta t$. At low optical depth, the transient peak increases as we increase optical depth because of many-atom collective enhancement, consistent with FID approximation. Moreover, the near-atomic resonance FID field has a narrow linewidth $\left(\gamma_{13} / \pi=6 \mathrm{MHz}\right)$ and thus is immune from the effect of finite rise and fall time. At high optical depth, in the form as optical precursors, the far-off-resonance frequency components contribute most due to their low absorption. When we increase the optical depth, the Sommerfeld and Brillouin saddle points move further away from the atomic resonance and closer to the filter cut frequency. As a result, the precursor peak transmission drops. In experiment, we measure the transient peak values with two different fall times. The shorter rise time of $3 \mathrm{~ns}$ is obtained using an electro-optical modulator. The 1-ns case is only a theoretical simulation. When the fall time becomes zero, the transmission peak saturates to unity as $\alpha_{0} L>5$. Our theory and experiment show that, in Fig. 10, the optical coherent transients excited by a resonant step pulse at low optical depth

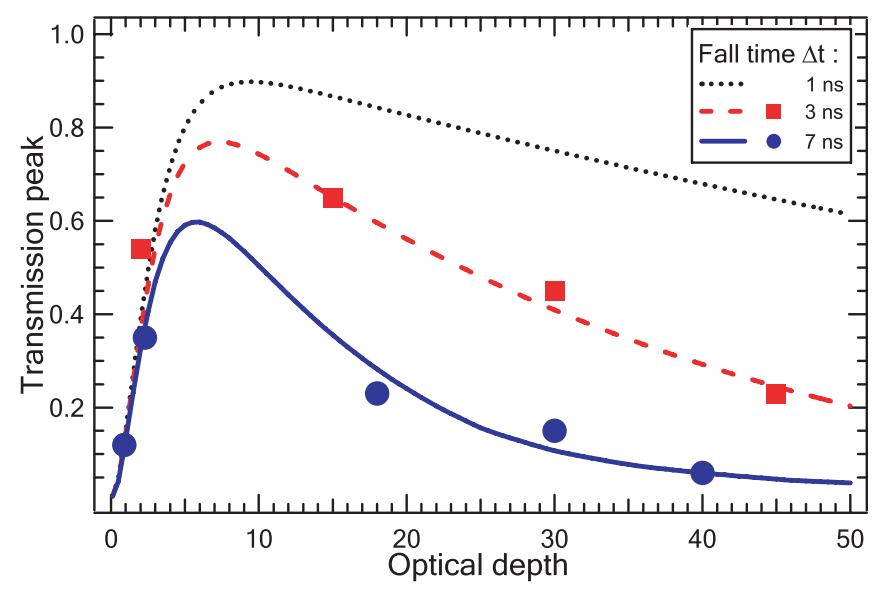

FIG. 10. (Color online) The transmission of the first transient peak after the falling edge as a function of optical depth in the twolevel system with the finite fall time of 1,3 , and $7 \mathrm{~ns}$. The probe laser is on resonance $\Delta \omega_{p}=0$. The circular and square markers are experimental data. The solid and dashed lines are theoretical curves. $\left(\alpha_{0} L<5\right)$ can be characterized as FID and those excited by a resonant step pulse at high optical depth $\left(\alpha_{0} L>5\right)$ as precursors.

\section{CONCLUSION}

In this article, we study optical coherent transients using time-domain atom-field coupled equations and frequencydomain linear dispersion theory, accompanied with a systematic experimental investigation in a wide parameter space. We find that both FID and precursors, which have been considered as two different phenomena, can be unified within a single theoretical frame, and they share the same origin: coherent transient responses from a dispersive medium to the sudden change of the external driving field. When the probe pulse is weak, both time- and frequency-domain approaches give a precise description for both FID and optical precursors. In the time domain, FID reflects the evolution of on-resonance atomic excitations that cannot disappear immediately, even without the driving field. On the other side, an optical precursor is the propagation of the leading edge of the step pulse that travels at the speed of light in vacuum because the atoms have finite response time-they cannot respond to the leading edge immediately. In the frequency domain, FID comes from the excitations on atomic absorptive resonances with narrow linewidth and is strongly coupled to the atomic medium, while precursors are contributed from the far-off-resonance wide spectral components that are weakly coupled to the medium and belong to ultrafast phenomena. In general, both contribute to optical transients. At low optical depth, the FID on-resonance excitations dominate, while at high optical depth, precursors dominate because of the large on-resonance absorption. With our cold atom apparatus, we measure optical transients from both the two-level absorber and EIT three-level system with the optical depth ranging from 0 to 45 .

At low optical depth, we obtain an approximate analytic expression for FID in the two-level system from both time- and frequency-domain approaches. We study FIDs with both onresonance and off-resonance external driving fields. We verify that FID excited by a weak field is indeed always on atomic resonance and decay with a time constant of $1 / \gamma_{13}$. This weak excitation and evolution can be well described by the linear dispersion theory, and it is not a spontaneous emission [43]. Optical pumping and power saturation effects at high probe powers are observed. The theory agrees well with experiment.

At high optical depth, we derive analytic expression for precursor and main field using hybrid-asymptotic analysis for on-resonance input probe pulse. The precursors, identical for both the two-level and EIT systems, following the leading edges, travel with a speed close to $c$. The main field is absorbed in a two-level system, while it is delayed in the EIT system without loss. We observe oscillatory features at the falling edge in the EIT system with a peak transmission of about $150 \%$, resulting from the interference between the precursor and the delayed main field. We have also studied optical precursors when the probe laser is off resonance.

By varying the optical depth from 0 to 45 , we observe the evolution from FID to precursors. On the FID side $\left(\alpha_{0} L<5\right)$, the transient field amplitude increases as we increase optical depth due to the collective enhancement. On the precursor 
side $\left(\alpha_{0} L>5\right)$, the transient field amplitude decreases with increasing optical depth because of the finite rise and fall time. Observation of such an evolution provides a strong evidence for our unified theory and explanation. Researchers of both FID and precursors have contributed to the field, even though they have not referenced each others' work. We therefore notice that the optical transient in Toyoda's experiment with an optical depth of 9 was called an FID [33], but can also be connected to precursors.

\section{ACKNOWLEDGMENTS}

The work was supported by the Hong Kong Research Grants Council (Project No. HKUST600809 and DAG08/09.SC02) and William Mong Clusters Laboratory. Some equipment used in the experiment were provided by the Joyce M. Kuok Lasers and Photonics Laboratory.

\section{APPENDIX A: DERIVATION OF FID AT LOW OPTICAL DEPTH}

The FID field solution, given in Eq. (10), is valid for small optical depth because the absorption has been neglected. To take into account the loss and absorption in the two-level medium at a finite optical depth, here we consider the induced electric dipole from the FID field as a perturbation. The total induced electric dipole can be written as

$$
P(z, t)=P_{0}(t)+P_{\mathrm{FID}}(z, t),
$$

where the first term has been given in Eq. (8). Because the FID field has a narrow linewidth of $\gamma_{13}$ [Eq. (10)], we have approximately $P_{\mathrm{FID}}(z, t) \simeq \varepsilon_{0} \chi_{0} E_{\mathrm{FID}}(z, t)$, where $\chi_{0}=\chi(0)$ is the on-resonance linear susceptibility. Therefore, for $t>0$, the slowly varying envelope equation (4) can be approximated as

$$
\begin{aligned}
& {\left[\frac{\partial}{\partial z}+\frac{1}{c} \frac{\partial}{\partial t}\right] E_{\mathrm{FID}}(z, t)} \\
& \quad \simeq \frac{i k_{p 0}}{2 \varepsilon_{0}}\left[P_{0}(t)+\varepsilon_{0} \chi_{0} E_{\mathrm{FID}}(z, t)\right] .
\end{aligned}
$$

From Eq. (16), the on-resonance linear susceptibility of a twolevel system is $\chi_{0}=i \alpha_{0} / k_{p 0}$. Then we rewrite Eq. (A2) as

$$
\frac{\partial E_{\mathrm{FID}}(z, t)}{\partial z}+\left[\frac{1}{c} \frac{\partial}{\partial t}+\frac{\alpha_{0}}{2}\right] E_{\mathrm{FID}}(z, t) \simeq \frac{i k_{p 0}}{2 \varepsilon_{0}} P_{0}(t) .
$$

When the FID field has a narrow linewidth and its envelope varies slowly, that is, $\frac{1}{c} \frac{\partial}{\partial t} \ll \frac{\alpha_{0}}{2}$, one can drop the term $\frac{1}{c} \frac{\partial}{\partial t}$. In our cold atom system with $\gamma_{13}=2 \pi \times 3 \mathrm{MHz}$, this condition is always held. For example, taking a typical optical depth $\alpha_{0} L=1$ and $L=1.5 \mathrm{~cm}$, we estimate $\frac{L}{c} \frac{\partial}{\partial t} E_{\mathrm{FID}} \sim$ $\frac{L}{c} \gamma_{13} E_{\mathrm{FID}}=10^{-5} E_{\mathrm{FID}} \ll \frac{\alpha_{0} L}{2} E_{\mathrm{FID}}$. Under the preceding approximations, we obtain the following equation for the slowly varying FID field envelope:

$$
\left[\frac{\partial}{\partial z}+\frac{\alpha_{0}}{2}\right] E_{\mathrm{FID}}(z, t) \simeq \frac{i k_{p 0}}{2 \varepsilon_{0}} P_{0}(t),
$$

with the boundary condition $E_{\mathrm{FID}}(z=0, t)=0 . \alpha_{0} / 2$ is the on-resonance loss. The forward-wave Green function of the preceding equation is

$$
G\left(z-z^{\prime}\right)=e^{-\frac{\alpha_{0}\left(z-z^{\prime}\right)}{2}} \Theta\left(z-z^{\prime}\right) .
$$

Thus, the FID field at the medium output surface $(z=L)$ can be obtained:

$$
\begin{aligned}
E_{\mathrm{FID}}(L, t) & =\frac{i k_{p 0}}{2 \varepsilon_{0}} \int_{0}^{L} G\left(L-z^{\prime}\right) P_{0}(t) d t^{\prime} \\
& =\frac{i k_{p 0} L}{2 \varepsilon_{0}} P_{0}(t) e^{-\alpha_{0} L / 4} \frac{\sinh \left(\alpha_{0} L / 4\right)}{\alpha_{0} L / 4} \\
& \simeq \frac{i k_{p 0} L}{2 \varepsilon_{0}} P_{0}(t) e^{-\alpha_{0} L / 4} .
\end{aligned}
$$

Instead of totally ignoring the medium absorption as Eq. (10), an absorption factor $e^{-\alpha_{0} L / 4}$, with the average absorption length equivalent to $L / 2$, is taken into account. At the low limit of $\alpha_{0} L$, Eq. (A4) can be approximated as $E_{\mathrm{FID}}(L, t) / L \simeq$ $\frac{i \omega_{p}}{2 c \varepsilon_{0}} P_{0}(t)$.

\section{APPENDIX B: OPTICAL PRECURSOR AND MAIN FIELD AT HIGH OPTICAL DEPTH}

The spectrum of a step-on or step-off input pulse, $E_{0 \pm}(t)=$ $E_{0} \Theta( \pm t)$, is given by

$$
E_{0 \pm}(\omega)= \pm \frac{i E_{0}}{\omega}+\pi E_{0} \delta(\omega)
$$

At high optical depth, for far-off-resonance Sommerfeld and Brillouin saddle points, the EIT medium can be approximated as a two-level absorber. The two far-off-resonance saddle points can be obtained following $d \phi / d \omega=0$,

$$
\omega_{\mathrm{sp}}= \pm \xi(t)-i \gamma_{13}
$$

where $\xi(t)=\sqrt{\alpha_{0} L \gamma_{13} /(2 \tau)}$, with $\tau=t-L / c$. As $\tau \rightarrow 0$ or $\alpha_{0} L \rightarrow \infty$, the saddle points move infinitely far away from resonance. Following the asymptotic analysis used in Refs. [7-9,17], one can obtain an analytical expression for the SB precursor [24]:

$$
\begin{aligned}
E_{S B \pm}(t) \simeq & E_{0} \sqrt{\frac{2}{\pi \sqrt{2 \alpha_{0} L \gamma_{13} \tau}}} \\
& \times \cos \left(\sqrt{2 \alpha_{0} L \gamma_{13} \tau}-\pi / 4\right) \Theta(\tau) e^{-\gamma_{13} \tau} \\
\simeq & \pm E_{0} J_{0}\left(\sqrt{2 \alpha_{0} L \gamma_{13} \tau}\right) \Theta(\tau) e^{-\gamma_{13} \tau} .
\end{aligned}
$$

This shows that at high optical depth, the optical precursor in an EIT system is nearly identical to that in a two-level system. To properly take into account the finite rise (fall) time $\Delta t$, we approximately treat the rising and falling edges as a linear sweep of the field amplitude. Then the effect of finite rise (fall) time can be simulated by convolving Eq. (B3) with a square function with a length of $\Delta t$ :

$$
\tilde{E}_{S B \pm}(t)=\frac{1}{\Delta t} \int_{0}^{\Delta t} E_{S B \pm}\left(t-t^{\prime}\right) d t^{\prime} .
$$

Now we turn to the on-resonance main field. For the twolevel system, the main field is absorbed at high optical depth. For the EIT system, the delayed main field cannot be obtained using the same asymptotic method as for the precursor because 
of the singularity in Eq. (B1) at $\omega=0$ and $d^{2} \phi(\omega) / d \omega^{2} \simeq 0$. When the ground-state dephasing is negligible $\left(\gamma_{12} \approx 0\right.$ or $\left.\left|\Omega_{c}\right|^{2} \gg 4 \gamma_{12} \gamma_{13}\right)$ and the EIT transparency window is very narrow, we can extend the EIT linear susceptibility [Eq. (15)] up to the third order about $\omega$ : $\chi(\omega) \simeq \chi(0)+\chi^{\prime}(0) \omega+$ $\frac{1}{2} \chi^{\prime \prime}(0) \omega^{2}+\frac{1}{6} \chi^{\prime \prime \prime}(0) \omega^{3}$. Then the transfer function can be approximated as

$$
H(\omega) \simeq e^{-\gamma_{12} \tau_{g}} e^{-\omega^{2} /\left(2 a^{2}\right)} e^{i \omega \tau_{g}} e^{i \omega^{3} /\left(3 b^{3}\right)} .
$$

The term $e^{-\gamma_{12} \tau_{g}}$ stands for the finite loss caused by the ground-state dephasing $\gamma_{12}$. Equation (B6) characterizes the EIT transmission profile at high optical depth.
Its impulse response function can be expressed as a convolution:

$$
h(t)=\frac{a b}{\sqrt{2 \pi}} e^{-\gamma_{12} \tau_{g}} e^{-\frac{1}{2} a^{2}\left(t-\tau_{g}\right)^{2}} * \operatorname{Ai}(-b t) .
$$

$\mathrm{Ai}(b t)$ is an Airy function. Then the main field can be obtained by

$$
\begin{aligned}
& E_{M \pm}(t) \simeq E_{0} \Theta( \pm t) * h(t) \\
& \quad=\frac{E_{0}}{2} e^{-\gamma_{12} \tau_{g}}\left(1 \pm \operatorname{erf}\left[\frac{a\left(t-\tau_{g}\right)}{\sqrt{2}}\right]\right) * b \operatorname{Ai}(-b t) .
\end{aligned}
$$

As expected, the main field is delayed by $\tau_{g}$. The Airy function adds a small modulation on top of the main field and leads to the so-called "postcursor" [8].
[1] H. A. Lorentz, The Theory of Electrons (Dover, New York, 1952).

[2] A. Sommerfeld, Ann. Phys. 44, 177 (1914); L. Brillouin, ibid. 44, 203 (1914).

[3] L. Brillouin, Wave Propagation and Group Velocity (Academic Press, New York, 1960).

[4] K. E. Oughstun and G. C. Sherman, Electromagnetic Pulse Propagation in Causal Dielectrics (Springer-Verlag, Berlin, 1994).

[5] N. A. Cartwright and K. E. Oughstun, SIAM Rev. 49, 628 (2007).

[6] H. Jeong and U. L. Österberg, Phys. Rev. A 77, 021803(R) (2008).

[7] H. Jeong and S. Du, Phys. Rev. A 79, 011802(R) (2009).

[8] B. Macke and B. Segard, Phys. Rev. A 80, 011803(R) (2009).

[9] W. R. LeFew, S. Venakides, and D. J. Gauthier, Phys. Rev. A 79, 063842 (2009).

[10] J. Aaviksoo, J. Kuhl, and K. Ploog, Phys. Rev. A 44, R5353 (1991).

[11] S.-H. Choi and U. L. Österberg, Phys. Rev. Lett. 92, 193903 (2004).

[12] H. Jeong, A. M. C. Dawes, and D. J. Gauthier, Phys. Rev. Lett. 96, 143901 (2006).

[13] S. Du, P. Kolchin, C. Belthangady, G. Y. Yin, and S. E. Harris, Phys. Rev. Lett. 100, 183603 (2008).

[14] S. Du, C. Belthangady, P. Kolchin, G. Y. Yin, and S. E. Harris, Opt. Lett. 33, 2149 (2008).

[15] F. J. Lynch et al., Phys. Rev. 120, 513 (1960).

[16] P. Pleshko and I. Palocz, Phys. Rev. Lett. 22, 1201 (1969).

[17] E. Varoquaux, G. A. Williams, and O. Avenel, Phys. Rev. B 34, 7617 (1986).

[18] E. Falcon, C. Laroche, and S. Fauve, Phys. Rev. Lett. 91, 064502 (2003).

[19] R. R. Alfano, J. L. Birman, X. Ni, M. Alrubaiee, and B. B. Das, Phys. Rev. Lett. 94, 239401 (2005).

[20] T. M. Roberts, Phys. Rev. Lett. 93, 269401 (2004).

[21] B. Macke and B. Ségard, e-print arXiv:physics/0605039.

[22] S. E. Harris, Phys. Today 50, 36 (1997).
[23] M. Fleischhauer, A. Imamoglu, and J. P. Marangos, Rev. Mod. Phys. 77, 633 (2005).

[24] D. Wei, J. F. Chen, M. M. T. Loy, G. K. L. Wong, and S. Du, Phys. Rev. Lett. 103, 093602 (2009).

[25] R. G. Brewer and R. L. Shoemaker, Phys. Rev. Lett. 27, 631 (1971).

[26] R. G. Brewer and R. L. Shoemaker, Phys. Rev. A 6, 2001 (1972).

[27] K. L. Foster, S. Stenholm, and R. G. Brewer, Phys. Rev. A 10, 2318 (1974).

[28] D. Grischkowsky, M. M. T. Loy, and P. F. Liao, Phys. Rev. A 12, 2514 (1975).

[29] M. M. T. Loy, Phys. Rev. Lett. 36, 1454 (1976).

[30] P. F. Liao, J. E. Bjorkholm, and J. P. Gordon, Phys. Rev. Lett. 39, 15 (1977).

[31] F. Bloch, Phys. Rev. 70, 460 (1946).

[32] L. Allen and J. H. Eberly, Optical Resonance and Two-Level Atoms (Dover Publications, New York, 1987).

[33] K. Toyoda, Y. Takahashi, K. Ishikawa, and T. Yabuzaki, Phys. Rev. A 56, 1564 (1997).

[34] U. Shim, S. Cahn, A. Kumarakrishnan, T. Sleator, and J.-T. Kim, J. Appl. Phys. 41, 3688 (2002).

[35] H. J. Hartmann and A. Laubereau, J. Chem. Phys. 80, 4663 (1984).

[36] O. Avenel, E. Varoquaux, and G. A. Williams, Phys. Rev. Lett. 53, 2058 (1984).

[37] M. D. Crisp, Phys. Rev. A 1, 1604 (1970).

[38] H. Jeong and U. Österberg, J. Opt. Soc. Am. B 25, B1 (2008).

[39] R. P. Feynman, F. L. Vernon, and R. W. Hellwarth, J. App. Phys. 28, 49 (1957).

[40] M. D. Crisp, Phys. Rev. A 8, 2128 (1973).

[41] M. Wegener, Extreme Nonlinear Optics (Springer, Berlin/Heidelberg, 2005).

[42] J. D. Jackson, Classical Electrodynamics (Wiley, New York, 1975), 2nd ed.

[43] M. Afzelius, M. U. Staudt, H. de Riedmatten, C. Simon, S. R. Hastings-Simon, R. Ricken, H. Suche, W. Sohler, and N. Gisin, New J. Phys. 9, 413 (2007). 